\title{
Introducing a single secondary alcohol dehydrogenase into butanol-tolerant Clostridium acetobutylicum Rh8 switches ABE fermentation to high level IBE fermentation
}

\author{
Zongjie Dai ${ }^{1,2}$, Hongjun Dong ${ }^{2}$, Yan Zhu ${ }^{2,3}$, Yanping Zhang ${ }^{2}$, Yin Li2 ${ }^{2^{*}}$ and Yanhe Ma ${ }^{2}$
}

\begin{abstract}
Background: Previously we have developed a butanol tolerant mutant of Clostridium acetobutylicum Rh8, from the wild type strain DSM 1731. Strain Rh8 can tolerate up to $19 \mathrm{~g} / \mathrm{L}$ butanol, with solvent titer improved accordingly, thus exhibiting industrial application potential. To test if strain Rh8 can be used for production of high level mixed alcohols, a single secondary alcohol dehydrogenase from Clostridium beijerinckii NRRL B593 was overexpressed in strain Rh8 under the control of thl promoter.

Results: The heterogenous gene SADH was functionally expressed in C. acetobutylicum Rh8. This simple, one-step engineering approach switched the traditional ABE (acetone-butanol-ethanol) fermentation to IBE (isopropanolbutanol-ethanol) fermentation. The total alcohol titer reached $23.88 \mathrm{~g} / \mathrm{l}(7.6 \mathrm{~g} / \mathrm{l}$ isopropanol, $15 \mathrm{~g} / \mathrm{l}$ butanol, and $1.28 \mathrm{~g} / \mathrm{l}$ ethanol) with a yield to glucose of $31.42 \%$. The acid (butyrate and acetate) assimilation rate in isopropanol producing strain Rh8(psADH) was increased.

Conclusions: The improved butanol tolerance and the enhanced solvent biosynthesis machinery in strain Rh8 is beneficial for production of high concentration of mixed alcohols. Strain Rh8 can thus be considered as a good host for further engineering of solvent/alcohol production.
\end{abstract}

Keywords: Clostridium acetobutylicum, Butanol tolerance, Isopropanol-butanol-ethanol (IBE) fermentation, Secondary alcohol dehydrogenase, Acetone-butanol-ethanol (ABE) fermentation

\section{Background}

Clostridium acetobutylicum is a Gram-positive, sporeforming anaerobe which is mainly used for acetonebutanol-ethanol (ABE) fermentation [1]. To improve the yield, titer, and productivity of $\mathrm{ABE}$ fermentation, various engineering strategies have been developed. These includes engineering central carbon flux redistribution [2-6], engineering regulatory mechanism $[7,8]$, engineering phenotypic properties [9], and engineering butanol tolerance $[10,11]$. Of these strategies, engineering butanol tolerance has been demonstrated to be an effective approach to improve the productivity

\footnotetext{
* Correspondence: yli@im.ac.cn

${ }^{2}$ Institute of Microbiology, Chinese Academy of Sciences, No.1 West Beichen Road, Chaoyang District, Beijing 100101, China

Full list of author information is available at the end of the article
}

of $\mathrm{ABE}$ fermentation. For instance, introducing chaperon proteins groESL into C. acetobutylicum ATCC 824 [10] or introducing a glutathione biosynthetic pathway into C. acetobutylicum DSM 1731 [11] all resulted in an improved butanol tolerance and solvent productivity.

Improving butanol tolerance by random, nonrational engineering strategies can also lead to improved ABE productivity. Clostridium strain SA-1, a derivative of Clostridium beijerinckii ATCC 35702 obtained by serial enrichment can grow in the presence of $15 \mathrm{~g}$ butanol/liter and could produce 5-14\% higher concentration of butanol in corn broth than that of the wild-type strain [12]. With the same method, Clostridium strain G1 showed a $40 \%$ increase butanol tolerance and a $17 \%$ improvement in the titer

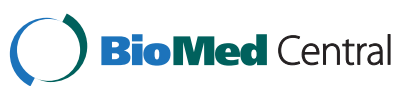

(c) 2012 dai et al.; licensee BioMed Central Ltd. This is an Open Access article distributed under the terms of the Creative Commons Attribution License (http://creativecommons.org/licenses/by/2.0), which permits unrestricted use, distribution, and reproduction in any medium, provided the original work is properly cited. 
of butanol [13]. C. acetobutylicum EA 2018 has greater capability of solvent production, than the wild type strain C. acetobutylicum ATCC 824, especially for butanol, which was generated through butanol resistance screening of $\mathrm{N}$-methyl-N-nitro-N-nitrosoguanidine treated Clostridium strain [14]. All these strains were obtained through native evolution or traditional mutagenesis. In our previous study, a butanol tolerant $C$. acetobutylicum mutant Rh8 was obtained by chemical mutagenesis and genome shuffling. This mutant showed a $46 \%$ improved butanol and $20 \%$ improved solvent titer, compared to that of the wild type strain DSM 1731 [15]. Characterization of strain Rh8 on the cytoplasmic and membrane proteome level revealed that strain Rh8 has developed a more stabilized membrane structure, a cost-efficient energy metabolism strategy, an earlier initiation of stress response mechanism, and strengthened solvent formation pathway $[15,16]$. These comprehensive characterizations suggest that strain Rh 8 might be considered as an interesting host for further improvement of solvent productivity.

Recently, an alternative strategy to improve the costeffectiveness for ABE fermentation was developed [17]. This strategy employs a secondary alcohol dehydrogenase [18] to convert acetone in $\mathrm{ABE}$ fermentation into a more expensive product isopropanol, thus switching ABE fermentation into IBE (isopropanol-butanol-ethanol) fermentation. Expression of a $S A D H$ gene from $C$. beijerinckii NRRL B593 into C. acetobutylicum ATCC 824 resulted in production of mix solvents $(0.1 \mathrm{~g} / \mathrm{l}$ acetone, $3.1 \mathrm{~g} / \mathrm{l}$ isopropanol, $7.3 \mathrm{~g} / \mathrm{l}$ butanol). The authors further constructed a finely engineered strain, where a synthetic acetone operon $(a d c, c t f A, c t f B)$ and $s A D H$ were overexpressed in a buk gene deletion mutant of C. acetobutylicum ATCC 824. The resulted engineered strain produced $20.4 \mathrm{~g} / \mathrm{l}$ mixed alcohols (4.4 g/l isopropanol, $14.1 \mathrm{~g} / \mathrm{l}$ butanol, and $1.9 \mathrm{~g} / \mathrm{l}$ ethanol) in batch fermentation, with a yield to glucose of $0.3 \mathrm{~g} / \mathrm{g}$.

For a strain with industrial application potential, it is desirable to minimize the genetic manipulations. The well characterized butanol-tolerant mutant Rh8 strain showed a robust cellular structure and strengthened solvent biosynthesis, therefore can be considered as a good start point for engineering strains of industrial relevance. We investigated the potential of strain Rh8 using switch from ABE fermentation to IBE fermentation as a model. Surprisingly, we found simply introducing the $s A D H$ gene from C. beijerinckii NRRL B593 into strain Rh8 under the control of thl promoter resulted in a complete conversion of acetone into isopropanol, as well as a higher yield and higher titer of mixed alcohol in batch fermentations. The genetic operability and the capability of producing high level mix alcohols demonstrated that strain Rh8 is a potential good host for production of bio-based chemicals.

\section{Results}

\section{Functional expression of secondary alcohol} dehydrogenase in C. acetobutylicum Rh8

To test the genetic manipulation feasibility of strain Rh8, both methylated and unmethylated shuttle vector pIMP1 were transformed into this mutant strain. The results showed that strain Rh8 could accept the methylated plasmid with normal transformation efficiency $\left(10^{4}\right.$ transformants per $\mu \mathrm{g}$ DNA). As to unmethylated plasmid, no transformant could be found on erythromycin plate. It means methlyating plasmid before transformation is still a necessary step for this strain. Previous work showed that the secondary alcohol dehydrogenase (encoded by $s A D H$ ) from $C$. beijerinckii NRRL B593 could convert acetone into isopropanol in C. acetobutylicum ATCC 824 [17]. In that study, a late expression adc promoter was used, which might be the reason why there was little residual acetone still present in the culture medium. We cloned the secondary alcohol dehydrogenase gene $(s A D H)$ from C. beijerinckii NRRL B593 into pIMP1 under the control of the thl promoter. The methylated plasmid psADH was transformed into C. acetobutylicum Rh8. The overexpression of $s A D H$ gene was confirmed through SDS-PAGE analysis (Figure 1b). The cellular catalytic activity was detected by adding $5 \mathrm{~g} / \mathrm{l}$ acetone into the RCM culture medium at exponential growth stage, then measuring the production of isopropanol with different time course. In contrast with the control strain, C. acetobutylicum Rh8(psADH) could completely convert the added acetone into isopropanol in 18 hours (Figure 1c). The results suggested that heterologous $s A D H$ gene was functional in C. acetobutylicum Rh8.

\section{Fermentation profiles of C. acetobutylicum Rh8(psADH) in CGM culture medium}

The Clostridium strain Rh8 has a stronger capability for ABE production compared with the wild type strain DSM $1731[15,16]$. The strain also showed a stronger IBE production capability, when introducing the secondary alcohol dehydrogenase (Table 1). Batch fermentations were performed in CGM culture medium under $\mathrm{pH}$ control $(\geq 5.0)$ (Figure 2). The results showed that $C$. acetobutylicum $\mathrm{Rh} 8(\mathrm{psADH})$ produced $7.6 \pm 0.1 \mathrm{~g} / \mathrm{l}$ isopropanol, $15 \pm 0.1 \mathrm{~g} / \mathrm{l}$ butanol and $1.28 \pm 0.1 \mathrm{~g} / \mathrm{l}$ ethanol (total solvent yield to glucose, $31.4 \%$ ) after $48 \mathrm{~h}$ fermentation. Residual concentrations of acetate and butyrate were $0.5 \pm 0.1 \mathrm{~g} / \mathrm{l}$ and $2.2 \pm 0.2 \mathrm{~g} / \mathrm{l}$, respectively. Notably, GC-MS data showed that there was almost no 

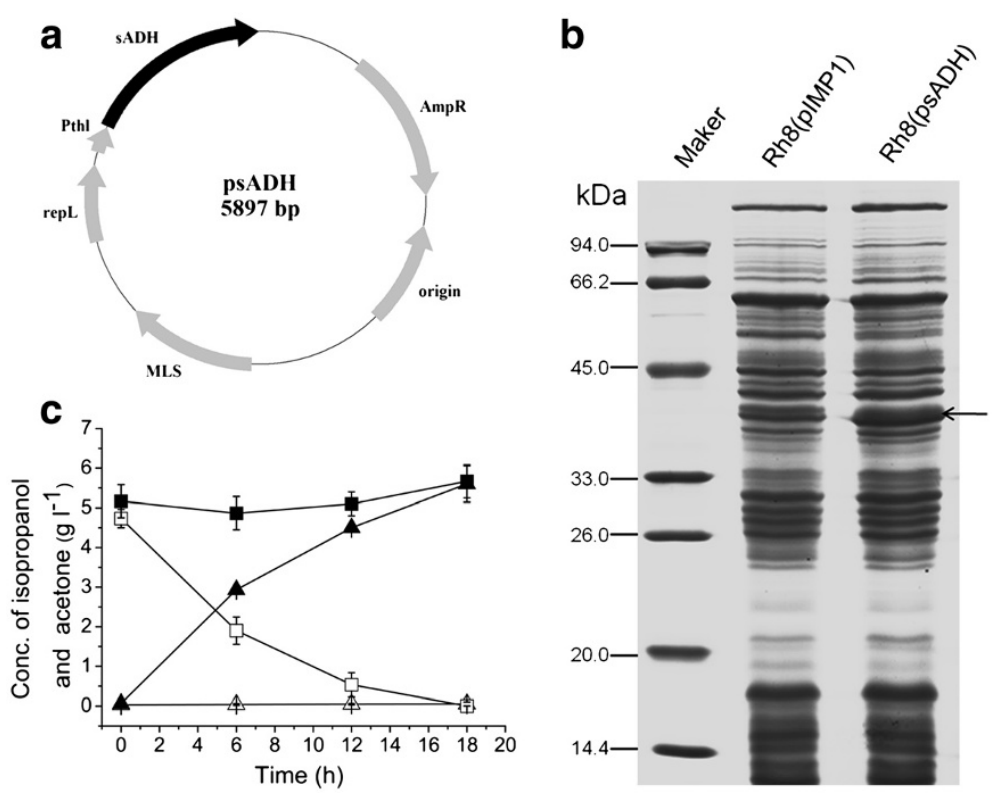

Figure 1 Overexpression of $s A D H$ gene in C. acetobutylicum Rh8. (a) Plasmid for expression of SADH gene in C. acetobutylicum Rh8. (b) SDSPAGE analysis of overexpressing SADH gene in strain Rh8. The overexpressed protein sADH (theoretical molecular weight $37.65 \mathrm{kDa}$ ) is indicated by the arrow on the right. Strain Rh8(pIMP1) is the control strain containing empty vector. (c) Detection of sADH acitivty. C. acetobutylicum Rh8 (psADH) and C. acetobutylicum Rh8(pIMP1) cells were cultured in RCM culture medium adding $5 \mathrm{~g} / \mathrm{l}$ acetone when cells grow into exponential stage $\left(\mathrm{OD}_{600}=0.8\right)$. The concentrations of acetone (solid squares/open squares) and isopropanol (open triangle/solid squares) in strain Rh8 (pIMP1)/Rh8(psADH) were determined by HPLC.

Table 1 Comparison of major product profiles in $\mathrm{pH}$-controlled $(\mathrm{pH} \geq 5.0)$ batch fermentations of $\mathrm{C}$. acetobutylicum strains

\begin{tabular}{|c|c|c|c|c|}
\hline \multirow[t]{2}{*}{ Characteristics and products } & \multicolumn{4}{|l|}{ Strains } \\
\hline & Rh8(pIMP1) & Rh8(psADH) & 1731(pIMP1) & 1731 (psADH) \\
\hline \multicolumn{5}{|l|}{ Butanol } \\
\hline & $17.3 \pm 0.2$ & $15 \pm 0.1$ & $14 \pm 0.2$ & $12 \pm 0.1$ \\
\hline \multirow[t]{2}{*}{ Isopropanol } & ND & & ND & \\
\hline & & $7.6 \pm 0.1$ & & $7.15 \pm 0.1$ \\
\hline \multirow[t]{2}{*}{ Acetone } & & ND & & ND \\
\hline & $7.5 \pm 0.1$ & & $4.44 \pm 0.1$ & \\
\hline \multicolumn{5}{|l|}{ Ethanol } \\
\hline & $2.72 \pm 0.1$ & $1.28 \pm 0.1$ & $1.8 \pm 0.1$ & $1.5 \pm 0.1$ \\
\hline \multirow[t]{2}{*}{ Butyrate } & & $2.2 \pm 0.2$ & & \\
\hline & $0.93 \pm 0.1$ & & $1.8 \pm 0.2$ & $2.1 \pm 0.2$ \\
\hline \multirow[t]{2}{*}{ Acetate } & & $0.5 \pm 0.1$ & & \\
\hline & $0.82 \pm 0.2$ & & $3.5 \pm 0.2$ & $2.8 \pm 0.2$ \\
\hline \multicolumn{5}{|l|}{ Total solvent } \\
\hline & $27.52 \pm 0.4$ & $23.88 \pm 0.3$ & $20.24 \pm 0.4$ & $20.65 \pm 0.3$ \\
\hline $\mathrm{IB}$ or $\mathrm{AB}$ ratio $^{\mathrm{a}}$ & 0.9 & 0.95 & 0.91 & 0.93 \\
\hline Solvent yield & 0.336 & 0.314 & 0.277 & 0.287 \\
\hline
\end{tabular}

a (Isopropanol + butanol)/total solvent (g/g) or (acetone + butanol)/total solvent. 


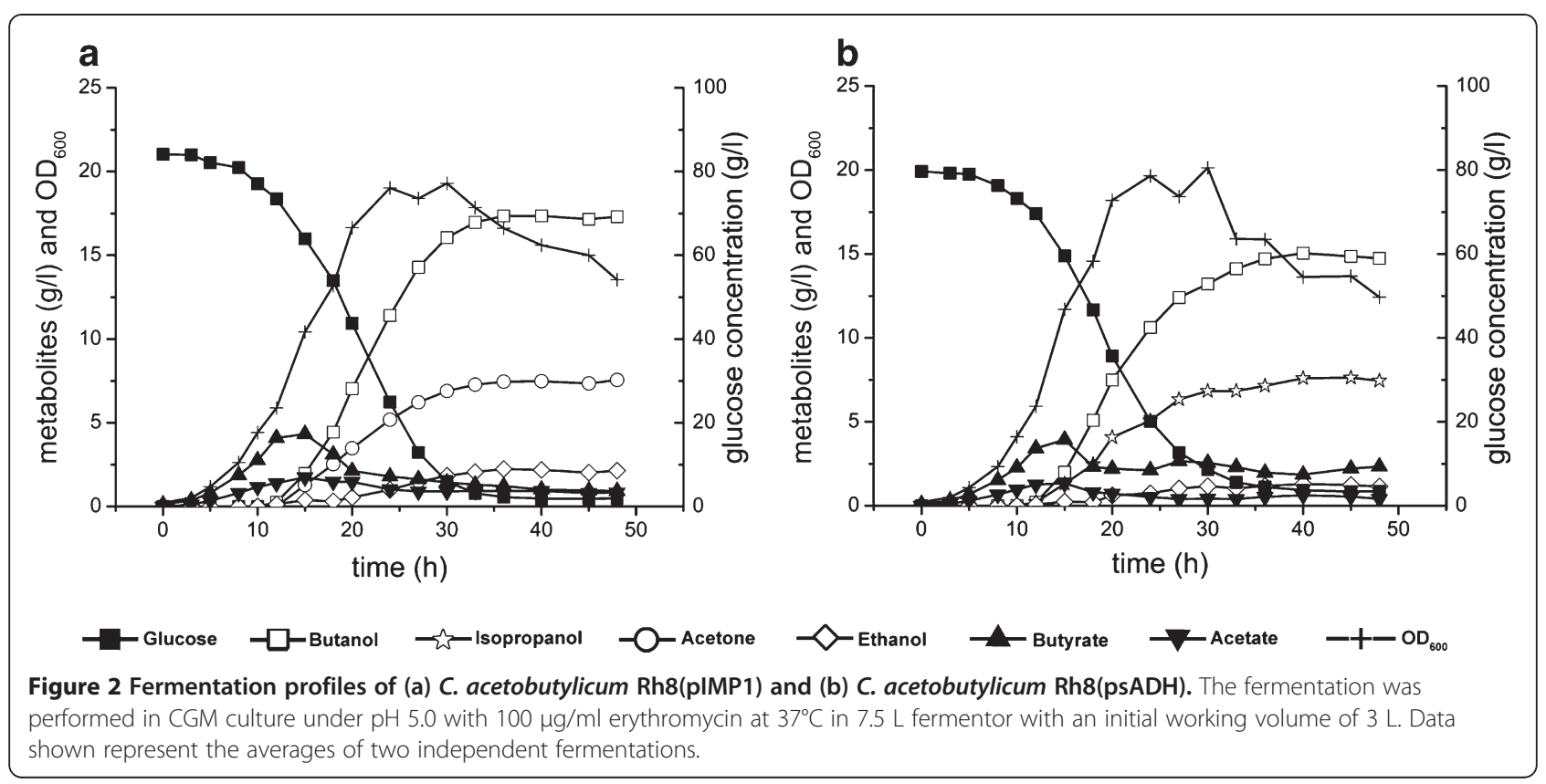

acetone $(<0.02 \mathrm{~g} / \mathrm{l})$ in culture medium (Figure 3$)$. The control strain $\mathrm{Rh} 8$ (pIMP1) produced $7.5 \pm 0.1 \mathrm{~g} / \mathrm{l}$ acetone, $17.3 \pm 0.2 \mathrm{~g} / \mathrm{l}$ butanol, and $2.72 \pm 0.1 \mathrm{~g} / \mathrm{l}$ of ethanol (total solvent yield to glucose, 33.6\%). The amount of residual acetate and butyrate were $0.82 \pm 0.2 \mathrm{~g} / \mathrm{l}$ and $0.93 \pm 0.1 \mathrm{~g} / \mathrm{l}$. The maximum biomass of strain $\mathrm{Rh} 8$ (psADH) was $6.03 \pm 0.06$ which was slightly higher than that of control strain $(5.79 \pm 0.06)$. The ratio of isopropanol and butanol to total solvent of strain $\mathrm{Rh} 8$ (psADH) (0.95) was higher than that of control strain (0.9) (Table 1).
Overexpression of the secondary alcohol dehydrogenase increases the acid assimilation rate and redistributes the reducing equivalent in C. acetobutylicum $\mathrm{Rh} 8$

The secondary alcohol dehydrogenase introduced into strain Rh8 is an NADPH dependent enzyme. To better understand the metabolic shift from ABE production to IBE production when overexpressing the secondary alcohol dehydrogenase, metabolic flux analysis (MFA) was performed to compare the flux profiles of central metabolic pathways between C. acetobutylicum Rh8 (pIMP1) and C. acetobutylicum Rh8(psADH) (Figure 4).

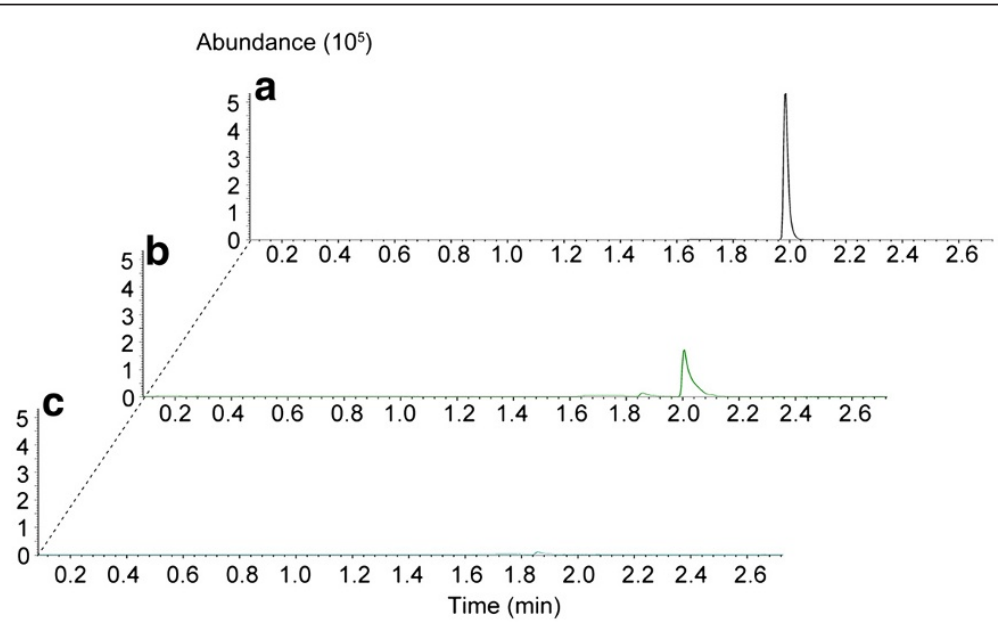

Figure 3 GC-MS analysis the acetone in culture broth of C. acetobutylium Rh8(pIMP1) and C. acetobutylicum Rh8(psADH). (a) The standard retention time of acetone was about at $2.0 \mathrm{~min}$. (b) Detection of acetone in the culture broth of C. acetobutylicum Rh8(pIMP1). (c) Detection of residual acetone in the culture broth of C. acetobutylicum Rh8(psADH). 


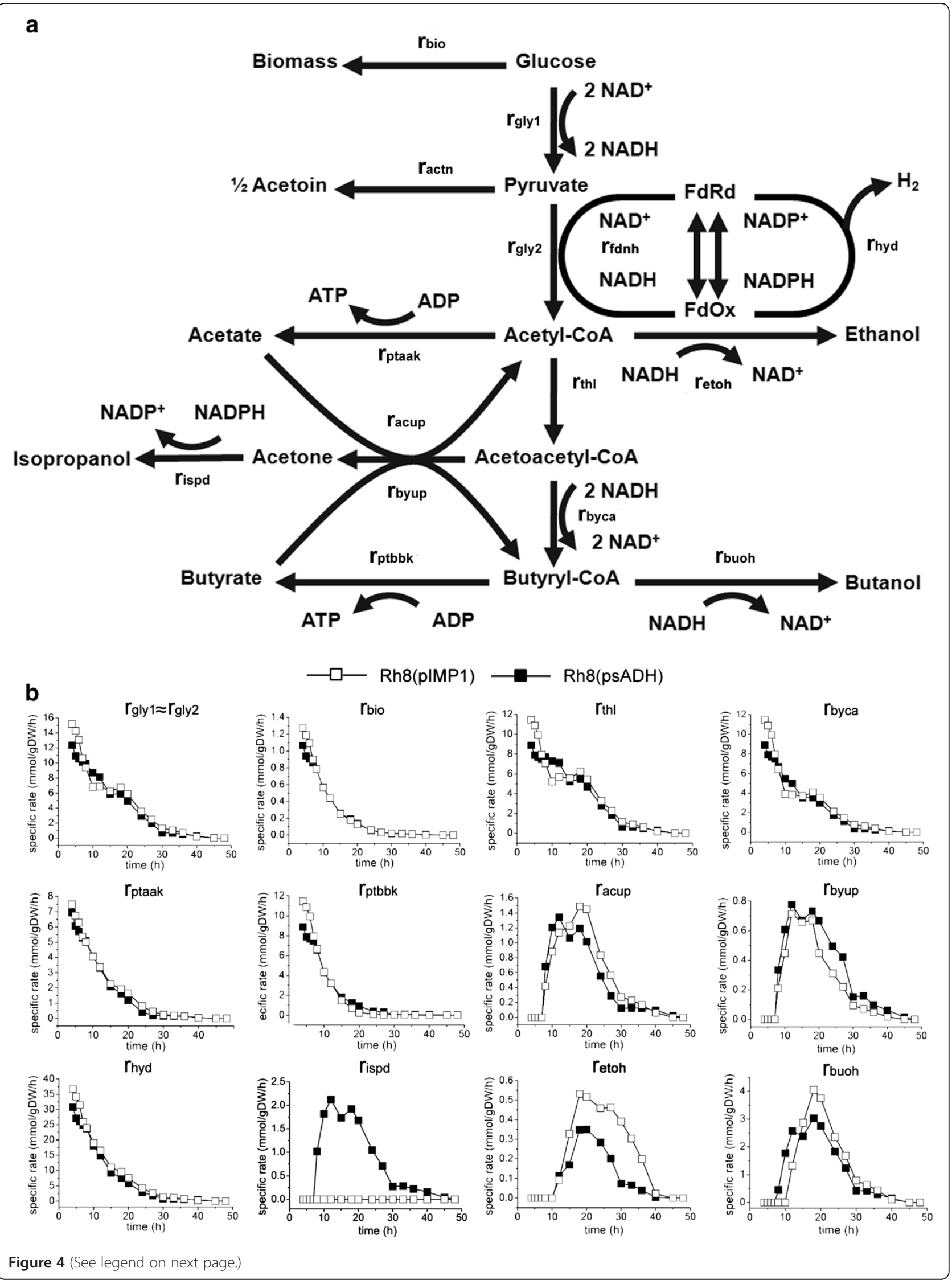


MFA showed that the two strains have similar acid (acetate and butyrate) production rate in the first 20 hours of fermentation, and there was almost no acid production afterwards. However, the acetate assimilation rate of $\mathrm{Rh} 8(\mathrm{psADH})$ was higher than that of the control strain from 8 to $15 \mathrm{~h}$, and the butyrate assimilation rate of strain Rh8(psADH) was also higher. This suggested that the extended reaction from acetone to isopropanol increased the acid assimilation rate.

The growth rates (rbio) and glucose consumption rates (rgly1 and rgly2) of two strains were almost the same, indicating that expressing the $s A D H$ gene under the thl promoter did not introduce metabolic burden. The reactions of consuming reducing equivalent include rhyd, rthl, rbyca, rispd, retoh and rbuoh. Although the enzyme that we introduced is NADPH dependent, there were no significant differences on hydrogen production (rhyd) and on the flux from acetyl-CoA to butyryl-CoA (rthl, rbyca) between these two strains. However, the butanol and ethanol production strength (rbuoh, retoh) of strain Rh8(psADH) was lower than that of the control strain Rh8(pIMP1). This suggests that introducing the $s A D H$ gene leads to the redistribution of reducing equivalent among alcohols (isopropanol, butanol, and ethanol).

\section{Discussion}

C. acetobutylicum Rh8 is a butanol-tolerant mutant with higher solvent production capability, which could convert 7-8 g/l acetone to isopropanol completely when overexpressing the secondary alcohol dehydrogenase under the control of thl promoter. Recently the $s A D H$ gene was integrated into Clostridium acetobutylicum chromosome under the control of thl promoter [19]. However, there was still $0.9 \mathrm{~g} / \mathrm{l}$ residual acetone present in the medium, presumably due to the low activity of the secondary alcohol dehydrogenase, as only one copy of $s A D H$ was introduced. Another group expressed this gene with adc promoter in Clostridium acetobutylicum ATCC 824, using pIMP1 as a based vector. A small amount of acetone $(<0.1 \mathrm{~g} / \mathrm{l})$ could be detected by GC in culture broth and the total residual concentration of acetone was amplified to about $1 \mathrm{~g} / \mathrm{l}$ when in situ gasstripping system was integrated with the fermentation [17]. The adc promoter was active during exponential growth, but maximal transcription from the adc promoter was reached in the exponential-growth later phase. Expression of the acetoacetate decarboxylase gene was turned off $2 \mathrm{~h}$ after reaching its maximum [20]. It means the expression of $s A D H$ gene would also turn off with the $a d c$ promoter. In this case, the residual enzyme would not be enough to convert the residual acetone to isopropanol. It is may be the reason why there was residual acetone in culture broth. We employed the most sensitive GC-MS to detect the presence of acetone in the culture broth of strain Rh8 (psADH). The data showed that acetone was below the trace amount $(<0.02 \mathrm{~g} / \mathrm{l})$ that the GC-MS could detect. This suggests that increasing the activity of the secondary alcohol dehydrogenase by strongly and perpetually expressing the $s A D H$ gene is a prerequisite to completely converting acetone to isopropanol.

Our previous work on the comparative analysis of cytoplasm proteome of strain DSM 1731 and Rh8 showed that ten proteins related to solvent formation, which are known to increase expression at the onset of solventogenesis, were differently expressed. Seven out of ten proteins (THL, AdhE1, CtfA/B, Adc, BdhA/B) in strain Rh8 that are involved in acetone and butanol production, significantly upregulated in acidogenesis, and the expression of these proteins were further increased in solventogenesis. While in the wild type strain DSM 1731, the expression of these seven proteins were only upregulated when cells entered solventogenesis. This might be the key why strain Rh8 produces higher level of solvents than that of the wild type DSM 1731. Notably, the expression level of acetone operon ( $a d c-c t f A-c t f B)$ in strain Rh8, which is necessary for isopropanol production, was upregulated by a fold of 2.7 in acidogenesis compared with strain DSM 1731 [15]. We believe the significant upregulation of these solvent formation proteins contributed to strong capability of producing isopropanol and butanol by strain Rh8(psADH).

The production of butanol and ethanol by strain Rh8 (psADH) showed a little decrease as compared to the control strain. This might be due to that the production of isopropanol competes with the production of butanol and ethanol for the reducing equivalent $(\mathrm{NADH}$ and $\mathrm{NADPH}$ ). In C. acetobutylicum pyruvate-ferredoxin oxidoreductase could convert pyruvate into acetyl-CoA and carbon dioxide, producing reduced ferredoxin. This reduced ferredoxin could provide proton for production of NADPH, NADH, or hydrogen [21]. This is the major source for NADPH generation in C. acetobutylicum [22]. Nevertheless, no remarkable difference on the metabolic flux from glucose to acetyl-CoA (rgly1, rgly2) was found 
between strain Rh8(psADH) and strain Rh8(pIMP1). This suggests that the reducing equivalent produced by these two strains is in a similar amount. Since the secondary alcohol dehydrogenase introduced into strain $\mathrm{Rh} 8$ is an NADPH-dependent enzyme [18], isopropanol production will consume more reducing equivalent compared with strain Rh8(pIMP1). Therefore, it's not surprising that the flux towards the NADH-dependent production of ethanol and butanol decreased, since the flux of another reducing equivalent consuming pathways (rthl, rbyca, rhyd) of these two strains remained similar (Figure 3). Another implication from this study is that the central pathway from glucose to butyryl-CoA is quite robust, since introducing an NADPH-dependent secondary alcohol dehydrogenase did not lead to notable disturbance on the consumption or generation of reducing equivalent of this central pathway. This understanding might be helpful when designing new engineering strategy for C. acetobutylicum.

\section{Conclusions}

In this work, we introduce a secondary alcohol dehydrogenase into butanol-tolerant Clostridium strain Rh8. After 48 hours batch fermentation in glucose medium, this engineered strain could produce about $24 \mathrm{~g} / \mathrm{l}$ mixed alcohol with yield of $31.4 \%$. The potential of butanol-tolerant strain Rh8 was amplified by such simple metabolic engineering manipulation. Without downstream process, the total titer and yield of alcohol mixture was highest so far. It suggests that butanol tolerant strain is suitable host for further improving solvent or mixed alcohol production.

\section{Methods}

\section{Bacterial strains, plasmids and culture conditions}

All bacterial strains and plasmids used in this work are listed in Table 2. C. acetobutylicum Rh8, C. acetobutylicum DSM 1731 and C. beijerinckii NRRL B593 strains were stored in $15 \%$ (vol $/ \mathrm{vol}$ ) glycerol at $-80^{\circ} \mathrm{C}$. E. coli $\mathrm{DH} 5 \alpha$ was used for cloning and vectors maintenance. E. coli ER2275 bears the methylating plasmid pAN1 for methylation plasmids [23]. E. coli strains were grown aerobically at $220 \mathrm{rpm}$ and $37^{\circ} \mathrm{C}$ in liquid LB medium or on solidified LB medium supplementing with $1.5 \%$ agar. $C$. strains were grown in reinforced clostridial medium (RCM) [24] anaerobically at $37^{\circ} \mathrm{C}$. Ampicillin and erythromycin were added at concentration of $100 \mu \mathrm{g} / \mathrm{ml}$ and $50 \mu \mathrm{g} / \mathrm{ml}$ if it is necessary. Cell density was determined by a UV-visible spectrophotometer (UV-2802PC; Unico, Shanghai, China) with the optical density at $600 \mathrm{~nm}$. The dry cell weight (DCW) could be calculated from $\mathrm{OD}_{600}$ using an equation that $\mathrm{DCW}(\mathrm{g} / \mathrm{l})=0.3 \times \mathrm{OD}_{600}[25]$.

\section{DNA isolation, manipulation and transformation}

The total genomic DNA of C. beijerinckii NRRL B593 and all the plasmids DNA in E. coli were isolated using an E.Z.N.A Plasmid Extraction Kit and E.Z.N.A Bacterial DNA Isolation Kit. PCR products and DNA fragments were purified with E.Z.N.A Cycle Pure Kit

Table 2 Strains and plasmids used in this study

\begin{tabular}{|c|c|c|}
\hline Strains and plasmids & Relevant charactristics & Soure or reference \\
\hline \multicolumn{3}{|l|}{ Strains } \\
\hline C. acetobutylicum DSM 1731 & Wild type strain & DSMZ \\
\hline C. acetobutylicum Rh8 & The mutant of C. acetobutylicum DSM 1731 & {$[15]$} \\
\hline C. beijierinckii NRRL B593 & Contains $S A D H$ gene, wild type & NRRL \\
\hline C. acetobutylicum & sADH expressing strain, harboring psADH & This study \\
\hline Rh8(psADH) & Control strain harboring pIMP1 & This study \\
\hline \multicolumn{3}{|l|}{ C. acetobutylicum } \\
\hline \multicolumn{3}{|l|}{ Rh8(pIMP1) } \\
\hline E. coli JM109 & $\operatorname{rec} A 1 \mathrm{mcr} B+h s d R 17$ & Lab storage \\
\hline E. coli ER2275(pAN1) & Used for plasmid methylation before transformed into C. acetobutylicum & {$[26]$} \\
\hline \multicolumn{3}{|l|}{ Plasmids } \\
\hline pAN1 & $\phi 3 t l, p 15 a$ ori, $\mathrm{Cm}^{r}$ & {$[26]$} \\
\hline pIMP1 & The control plasmid, MLS', Ampr', shuttle vector of E. coli-C. acetobutylicum & {$[26]$} \\
\hline plTF & MLS', Ampr', pIMP1 derivative for fdh expression under thl promoter & {$[27]$} \\
\hline psADH & Used for $s A D H$ expression with th/ promoter & This study \\
\hline
\end{tabular}

Abbreviations: $\mathrm{Amp}^{\mathrm{r}}$, ampicillin resistance; $\mathrm{Cm}^{\mathrm{r}}$, chloramphenicol resistance; $\mathrm{MLS}^{\mathrm{r}}$, macrolide, lincosamide, and streptogramin $\mathrm{B}$ resistance; $\phi 3 \mathrm{tl}, \phi 3 \mathrm{TI}$ methyltransferase gene of Bacillus subtilis phage $\phi 3 \mathrm{Tl}$; thl, the promoter of thiolase gene in C. acetobutylicum; DSMZ, German Collection of Microorganisms and Cell Cultures, Braunschweig, Germany. NRRL, Agricultural Research Service Culture Collection, United State. 
(Omega Biotek Inc., Guangzhou, China). All the enzymes were obtained from New England Biolabs (Beijing, China), and used according to the manufacturer's protocols. E. coli ER2275 (pAN1) was used as host strain for methylating all plasmids before transforming them into C. acetobutylicum Rh8 and C. acetobutylicum DSM 1731. Methylated plasmids were electroporated into $C$. acetobutylicum by the method developed by Mermelstein [26].

\section{Construction of vector psADH}

The gene encoding second alcohol dehydrogenase was cloned from the genomic DNA of $C$. beijerinckii NRRL B593, using primers sADH-1: 5'-CGCGGATCCATG AAAGGTTTTGCAATGCTAGGTATTTAATAAGTT-3' and sADH-2: 5'-CCGGAATTCTTATAATATAACTAC TGCTTTAATTAAGTC-3'. The resulted fragment was ligated into pITF [27] vector via $B a m \mathrm{HI}$ and EcoRI sites to construct psADH (Figure 1a) in which $s A D H$ gene was epxressed under control of consititutive thl promoter [27].

\section{Detection of sADH enzyme activity}

When the cells were cultured into exponential stage of growth $\left(\mathrm{OD}_{600}=0.8\right)$, acetone was added into the broth at a concentration of $5 \mathrm{~g} / \mathrm{l}$. The concentrations of acetone and isopropanol in the broth were assayed every 6 hours $(0$ h, 6 h, 12 h, 18 h) using High Performance Liquid Chromatography (HPLC).

\section{Cell free extracts preparation and protein expression analysis}

The method used for protein expression assay in $C$. acetobutylicum was conducted as described previously [11] with slightly modification. The fresh cells were harvested by centrifugation and re-suspended in $1 \mathrm{ml}$ TE buffer ( $\mathrm{pH}$ 7.5). The cells were sonicated on ice for ten minutes using a Sonifier S-450D (Branson Ultrasonics Corp., Danbury, CT, USA) with the following protocol: $5 \mathrm{~s}$ sonication and $5 \mathrm{~s}$ intervals at $300 \mathrm{~W}$. Cell debris was removed by centrifugation $(12,000 \mathrm{~g}$ for $10 \mathrm{~min}$ at $4^{\circ} \mathrm{C}$ ). The protein concentration in the rest cell free extract was qualified by using an RC DC protein assay kit (Bio-Rad) with bovine serum albumin as a standard. The same concentration protein was mixed with SDSPAGE loading buffer and boiling for ten minutes. $10 \mu \mathrm{l}$ samples were applied to a SDS-PAGE (12\%) and visualized by Coomassie blue staining. The gel was scanned by Image Scanner 3 (GE Healthcare).

\section{Batch fermentation in CGM culture medium}

Batch fermentations were performed in Bioflo 110 fermentors (New Brunswick Scientific, Edison, NJ) containing $3.0 \mathrm{~L}$ (working volume) of CGM [28] with 10\% inoculum size, according to the fermentation method described in the literature [29], with slight modification. $8 \mathrm{ml}$ refined corn oil was used as antifoam and $100 \mu \mathrm{g} /$ $\mathrm{ml}$ erythromycin were added to the bioreactor together with $300 \mathrm{ml}$ seed cultures $\left(\mathrm{OD}_{600}=1.0\right)$. Experiments were duplicated and the presented data were the average values.

\section{High performance liquid chromatography (HPLC) analysis of metabolites}

The main fermentation products (glucose, butanol, ethanol, acetone, isopropanol, butyrate, acetate) were determined using an Agilent 1200 high performance liquid chromatography (Agilent Technologies, Santa Clara, CA) with injection at volumes of $10 \mu \mathrm{l}$. An aminex HPX-87 H organic acid analysis column $(7.8 \times 300 \mathrm{~mm})$ (Bio-Rad Laboratories, Inc, CA) was maintained at $15^{\circ} \mathrm{C}$ with $0.05 \mathrm{mM}$ sulfuric acid as mobile phase and at a flow rate of $0.5 \mathrm{ml} \mathrm{min}^{-1}$. A refractive index (RI) detector (Agilent) was used for signal detection.

\section{Gas chromatography-mass spectrometry (GC-MS) detection for acetone}

The residual acetone in culture broth was detected by GC-MS on a Agilent Technologies 6890 N GC-5973 N MSD. The GC was equipped with HP-5MS column (5\%-phenyl-methylpolysiloxane as stationary phase; $30 \mathrm{~m} \times 0.25 \mathrm{~mm}$ id $\times 25 \mu \mathrm{m}$ film thickness, Agilent Technologies, Palo Alto, CA, USA). Helium (>99.999\%) was used as carrier gas with a constant flow rate $1 \mathrm{ml} /$ min. A $0.2 \mu \mathrm{l}$ water samples was injected with split (100:1). The inject temperature was $150^{\circ} \mathrm{C}$ and the GCMS transfer line temperature was $280^{\circ} \mathrm{C}$, ion source $230^{\circ} \mathrm{C}$, quadrupole $150^{\circ} \mathrm{C}$. All compounds were analyzed with a nominal electron beam energy of $70 \mathrm{eV}$, and scan range was 15-75 amu. Following injection, the column temperature was held at $30^{\circ} \mathrm{C}$ for $5 \mathrm{~min}$, then increased from $30^{\circ} \mathrm{C}$ to $150^{\circ} \mathrm{C}$ at $20^{\circ} \mathrm{C} / \mathrm{min}$. Compounds were identified by comparing their retention times with those of authentic reference compounds and the spectra with that of mass spectral libraries NIST02 (Rev. D. 04.00, Agilent Technologies, Palo Alto, CA, USA). Compounds were quantified by their selected ion abundances (acetone, ion $\mathrm{m} / \mathrm{z} 43, \mathrm{~m} / \mathrm{z} 58$ ) relative to that of the standard.

\section{Metabolic flux analysis}

The specific rates were calculated based on B-spline fitting according to previously work [30]. A stoichiometric model of $C$. acetobutylicum was derived from previously works with slightly modification [31]. It contains 16 metabolites and 15 reactions (See Appendix A). Combining with relations between acids uptake rates, a nonlinear objective (Eq. 1) was minimized to find an 
optimal rate distribution. The first item of the equation is a sum of weighted squared residuals and the second is the constraint on acids. Coding and calculation was implemented by using GAMS 23.6 with LGO solver.

$$
\begin{aligned}
\min & : f \\
& =\left\|W^{-1} A \hat{r}-W^{-1} x\right\|^{2} \\
& +\left(r B Y U P[\text { acetate }]_{\mathrm{e}}-0.315 \text { rACUP }[\text { butyrate }]_{\mathrm{e}}\right)^{2}
\end{aligned}
$$

\section{Appendix A}

Pathway stoichiometry describing metabolism of solventogenic clostridia, as described previously [32]. Reversible reactions are indicated with ' $\leftrightarrow$ ' while irreversible reactions are indicated with ' $\rightarrow$ '.

ID

Formula

$$
\begin{aligned}
& \mathrm{r}_{\text {bio }}{ }^{\mathrm{a}} \text { : glucose }+0.873 \mathrm{NADH} \\
& +14.85 \mathrm{ATP} \leftrightarrow 6 \mathrm{biomass} \\
& \mathrm{r}_{\text {gly1 }}: \text { glucose } \rightarrow 2 \text { pyruvate }+2 \mathrm{NADH}+2 \mathrm{ATP} \\
& \mathrm{r}_{\text {gly2 }}: \text { pyruvate } \\
& \rightarrow \text { acetyl }-\mathrm{CoA}+\mathrm{CO} 2+\text { ReducedFerredoxin } \\
& \mathrm{r}_{\mathrm{actn} 2}: \text { pyruvate } \rightarrow \text { acetoin }+2 \mathrm{CO}_{2} \\
& r_{\text {etoh }}: \text { acetyl }-\mathrm{CoA}+2 \mathrm{NADH} \rightarrow \text { ethanol } \\
& \mathrm{r}_{\text {ptaak }}: \text { acetyl }-\mathrm{CoA} \leftrightarrow \text { acetate }+\mathrm{ATP} \\
& \mathrm{r}_{\text {thl2 }}: \text { acetyl }-\mathrm{CoA} \leftrightarrow \text { acetoacetyl }-\mathrm{CoA} \\
& \mathrm{r}_{\text {acup }} \text { : acetoacetyl }-\mathrm{CoA}+\text { acetate } \\
& \rightarrow \text { acetone }+\mathrm{CO}_{2}+\text { acetyl }-\mathrm{CoA} \\
& \mathrm{r}_{\text {byup }} \text { : acetoacetyl }-\mathrm{CoA}+\text { butyrate } \\
& \rightarrow \text { acetone }+\mathrm{CO}_{2}+\text { butyryl }-\mathrm{CoA} \\
& \mathrm{r}_{\text {byca }} \text { : acetoacetyl }-\mathrm{CoA}+2 \mathrm{NADH} \leftrightarrow \text { butyryl }-\mathrm{CoA} \\
& \mathrm{r}_{\text {ptbbk }}: \text { butyryl }-\mathrm{CoA} \leftrightarrow \text { butyrate }+ \text { ATP } \\
& \mathrm{r}_{\text {buoh }} \text { : butyryl }-\mathrm{CoA}+2 \mathrm{NADH} \rightarrow \text { butanol } \\
& \mathrm{r}_{\text {hyd }}: \text { ReducedFerredoxin } \rightarrow \mathrm{H}_{2} \\
& \mathrm{r}_{\mathrm{fdnh}}: \text { ReducedFerredoxin } \leftrightarrow \mathrm{NADH} \\
& \mathrm{r}_{\text {ispd }}{ }^{\mathrm{b}} \text { : acetone }+\mathrm{NADH} \leftrightarrow \text { isopropanol }
\end{aligned}
$$

In biomass equation, NADH has been used as the single pool of reducing equivalents though reaction rispd is thought as NADPH-dependent. Essentially, all ATP produced is assumed to be consumed through growth and non-growth maintenance requirements. ATP stoichiometry was set to be 14.85 based on biomass composition equation in genome-scale metabolic model of $C$. acetobutylicum published before [33].

${ }^{a}$ The flux rbio was used as a measure of both growth and lysis by dynamically shifting the stoichiometric coefficients of glucose and NADH depending on the direction of rbio. For positive values of rbio, the indicated coefficients were used. For negative values of rbio, the coefficients of glucose and NADH were changed to 0 .

b reaction catalyzed by $s A D H$ which is overexpressed in this work.

\section{Abbreviations}

ABE: Acetone-butanol-ethanol; CGM: Clostridial growth medium; GC-MS: Gas chromatography-mass spectrometry; HPLC: High performance liquid chromatography; IBE: Isopropanol-butanol-ethanol; RCM: Reinforced clostridial medium; RI: Refractive index; SADH: Secondary alcohol dehydrogenase; SDS-PAGE: Sodium dodecyl sulfate polyacrylamide gel electrophoresis; TE: Tris-ethylenediaminetetraacetic acid.

\section{Competing interests}

The authors have declared that no competing interests exist.

\section{Authors' contributions}

Conceived the experiments: ZD, HD, YZ, YL, YM. Performed the experiments: $Z D, H D$. Analyzed the data: ZD, YZ, HD. Contributed reagents/materials/ analysis tools: ZD, HD, YZ. Wrote the paper: ZD, HD, YL. All authors read and approved the final manuscript.

\section{Acknowledgements}

This work was supported by National High Technology Research and Development Program of China (863 program, 2011AA02A208), National Basic Research Program of China (973 program, 2011CBA00807), and Knowledge Innovation Program of the Chinese Academy of Sciences. Yin Li is supported by the Hundreds of Talents Program of the Chinese Academy of Sciences. Yanping Zhang is supported by the Knowledge Innovation Program of the Chinese Academy of Sciences (No.KSCX2-EW-Q-14). The authors are indebted to Professor Eleftherios Terry Papoutsakis (University of Delaware, USA) for his kind providing pIMP1 and E. coli ER2275 (pAN1). We also thank ARS (NRRL) Culture Collection for providing C. beijerinckii NRRL B593.

\section{Author details}

'Department of Biochemistry and Molecular Biology, University of Science and Technology of China, Hefei, China. Institute of Microbiology, Chinese Academy of Sciences, No.1 West Beichen Road, Chaoyang District, Beijing 100101, China. ${ }^{3}$ Graduate School of the Chinese Academy of Sciences, Beijing, China.

Received: 7 April 2012 Accepted: 28 June 2012

Published: 28 June 2012

\section{References}

1. Lutke-Eversloh T, Bahl H: Metabolic engineering of Clostridium acetobutylicum: recent advances to improve butanol production. Curr Opin Biotechnol 2011, 22:634-647.

2. Jiang Y, Xu C, Dong F, Yang Y, Jiang W, Yang S: Disruption of the acetoacetate decarboxylase gene in solvent-producing Clostridium acetobutylicum increases the butanol ratio. Metab Eng 2009, 11:284-291.

3. Harris LM, Desai RP, Welker NE, Papoutsakis ET: Characterization of recombinant strains of the Clostridium acetobutylicum butyrate kinase inactivation mutant: need for new phenomenological models for solventogenesis and butanol inhibition? Biotechnol Bioeng 2000, 67:1-11.

4. Mermelstein LD, Papoutsakis ET, Petersen DJ, Bennett GN: Metabolic engineering of Clostridium acetobutylicum ATCC 824 for increased solvent production by enhancement of acetone formation enzymeactivities using a synthetic acetone operon. Biotechnol Bioeng 1993, 42:1053-1060. 
5. Kuit W, Minton NP, Lopez-Contreras AM, Eggink G: Disruption of the acetate kinase (ack) gene of Clostridium acetobutylicum results in delayed acetate production. Appl Microbiol Biotechnol 2012, 94:729-741.

6. Lehmann D, Honicke D, Ehrenreich A, Schmidt M, Weuster-Botz D, Bahl H, Lutke-Eversloh T: Modifying the product pattern of Clostridium acetobutylicum : physiological effects of disrupting the acetate and acetone formation pathways. Appl Microbiol Biotechnol 2012, 94:743-754

7. Nair RV, Green EM, Watson DE, Bennett GN, Papoutsakis ET: Regulation of the sol locus genes for butanol and acetone formation in Clostridium acetobutylicum ATCC 824 by a putative transcriptional repressor. J Bacteriol 1999, 181:319-330.

8. Alsaker KV, Spitzer TR, Papoutsakis ET: Transcriptional analysis of spoOA overexpression in Clostridium acetobutylicum and its effect on the cell's response to butanol stress. J Bacterio/ 2004, 186:1959-1971.

9. Tracy SWJ Bryan P, Fast Alan G, Indurthi Dinesh C, Papoutsakis Eleftherios T: Clostridia: the importance of their exceptional substrate and metabolite diversity for biofuel and biorefinery applications. Curr Opin Biotechnol 2011, 23:1-18.

10. Tomas CA, Welker NE, Papoutsakis ET: Overexpression of groESL in Clostridium acetobutylicum results in increased solvent production and tolerance, prolonged metabolism, and changes in the cell's transcriptional program. Appl Environ Microbiol 2003, 69:4951-4965.

11. Zhu LJ, Dong HJ, Zhang YP, Li Y: Engineering the robustness of Clostridium acetobutylicum by introducing glutathione biosynthetic capability. Metab Eng 2011, 13:426-434

12. Lin YL, Blaschek HP: Butanol production by a butanol-tolerant strain of Clostridium acetobutylicum in extruded corn broth. App/ Environ Microbiol 1983, 45:966-973.

13. Soucaille P, Joliff G, Izard A, Goma G: Butanol tolerance and autobacteriocin production by Clostridium acetobutylicum. Curr Microbiol 1987, 14:295-299.

14. Hu S, Zheng H, Gu Y, Zhao J, Zhang W, Yang Y, Wang S, Zhao G, Yang S, Jiang W: Comparative genomic and transcriptomic analysis revealed genetic characteristics related to solvent formation and xylose utilization in Clostridium acetobutylicum EA 2018. BMC Genomics 2011, 12:93.

15. Mao S, Luo Y, Zhang T, Li J, Bao G, Zhu Y, Chen Z, Zhang Y, Li Y, Ma Y: Proteome reference map and comparative proteomic analysis between a wild type Clostridium acetobutylicum DSM 1731 and its mutant with enhanced butanol tolerance and butanol yield. J Proteome Res 2009, 9:3046-3061.

16. Mao SM, Luo YM, Bao GH, Zhang YP, Li Y, Ma YH: Comparative analysis on the membrane proteome of Clostridium acetobutylicum wild type strain and its butanol-tolerant mutant. Mol Biosyst 2011, 7:1660-1677.

17. Lee J, Jang YS, Choi SJ, Im JA, Song H, Cho JH, Seung do Y, Papoutsakis ET, Bennett GN, Lee SY: Metabolic engineering of Clostridium acetobutylicum ATCC 824 for isopropanol-butanol-ethanol fermentation. Appl Environ Microbiol 2011, 78:1416-1423.

18. Ismaiel AA, Zhu CX, Colby GD, Chen JS: Purification and characterization of a primary-secondary alcohol dehydrogenase from two strains of Clostridium beijerinckii. J Bacteriol 1993, 175:5097-5105.

19. Heap JT, Ehsaan M, Cooksley CM, Ng YK, Cartman ST, Winzer K, Minton NP. Integration of DNA into bacterial chromosomes from plasmids without a counter-selection marker. Nucleic Acids Res 2012, doi:10.1093/nar/gkr1321.

20. Feustel L, Nakotte S, Durre P: Characterization and development of two reporter gene systems for Clostridium acetobutylicum. Appl Environ Microbiol 2004, 70:798-803

21. Petitdemange $H$, Cherrier $C$, Bengone JM, Gay R: Study of the NADH and NADPH-ferredoxin oxidoreductase activities in Clostridium acetobutylicum. Can J Microbiol 1977, 23:152-160.

22. Amador-Noguez D, Feng XJ, Fan J, Roquet N, Rabitz H, Rabinowitz JD: Systems-level metabolic flux profiling elucidates a complete, bifurcated tricarboxylic acid cycle in Clostridium acetobutylicum. J Bacteriol 2010, 192:4452-4461.

23. Mermelstein LD, Papoutsakis ET: In vivo methylation in Escherichia coli by the Bacillus subtilis phage phi $3 \mathrm{~T}$ I methyltransferase to protect plasmids from restriction upon transformation of Clostridium acetobutylicum ATCC 824. Appl Environ Microbiol 1993, 59:1077-1081.

24. Hirsch A, Grinsted E: Methods for the growth and enumeration of anaerobic spore-formers from cheese, with observations on the effect of nisin. J DAIRY RES 1954, 21:101-110.
25. Assobhei O: El Kanouni A, Ismaili M, Loutfi M, Petitdemange H: Effect of acetic and butyric acids on the stability of solvent and spore formation by Clostridium acetobutylicum ATCC 824 during repeated subculturing. J Ferment Bioeng 1998, 85:209-212.

26. Mermelstein LD, Welker NE, Bennett GN, Papoutsakis ET: Expression of cloned homologous fermentative genes in Clostridium acetobutylicum ATCC 824. Nat Biotechnol 1992, 10:190-195.

27. Dong HJ, Zhang YP, Dai ZJ, Li Y: Engineering Clostridium strain to accept unmethylated DNA. PLoS One 2010, 5:e9038.

28. Wiesenborn DP, Rudolph FB, Papoutsakis ET: Thiolase from Clostridium acetobutylicum ATCC 824 and its role in the synthesis of acids and solvents. Appl Environ Microbiol 1988, 54:2717-2722.

29. Nair RV, Bennett GN, Papoutsakis ET: Molecular characterization of an aldehyde/alcohol dehydrogenase gene from Clostridium acetobutylicum ATCC 824. J Bacteriol 1994, 176:871-885.

30. Wang S, Zhu Y, Zhang Y, Li Y: Controlling the oxidoreduction potential of the culture of Clostridium acetobutylicum leads to an earlier initiation of solventogenesis, thus increasing solvent productivity. Appl Microbiol Biotechnol 2011, 93:1021-1030.

31. Desai RP, Nielsen LK, Papoutsakis ET: Stoichiometric modeling of Clostridium acetobutylicum fermentations with non-linear constraints. J Biotechnol 1999, 71:191-205.

32. Papoutsakis ET: Equations and calculations for fermentations of butyric acid bacteria. Biotechnol Bioeng 1984, 26:174-187.

33. Senger RS, Papoutsakis ET: Genome-scale model for Clostridium acetobutylicum: Part I. Metabolic network resolution and analysis. Biotechnol Bioeng 2008, 101:1036-1052.

doi:10.1186/1754-6834-5-44

Cite this article as: Dai et al:: Introducing a single secondary alcohol dehydrogenase into butanol-tolerant Clostridium acetobutylicum Rh8 switches ABE fermentation to high level IBE fermentation. Biotechnology for Biofuels 2012 5:44.

\section{Submit your next manuscript to BioMed Central and take full advantage of:}

- Convenient online submission

- Thorough peer review

- No space constraints or color figure charges

- Immediate publication on acceptance

- Inclusion in PubMed, CAS, Scopus and Google Scholar

- Research which is freely available for redistribution 